\title{
Biologically Inspired Grasp Planning Using Only Orthogonal Approach Angles
}

\author{
Eric Rombokas, Peter Brook, Joshua R Smith, and Yoky Matsuoka
}

\begin{abstract}
One approach to robotic grasping is to compute hand configurations, including contact locations, which would maximize a variety of measures of grasp quality. There is evidence that human-guided robotic grasps exhibit orthogonality of the wrist as a key feature. Orthogonality of the hand to the object frame or surfaces is often included in state-of-the art grasp synthesis algorithms, but here we present a systematic study of the efficacy of orthogonality alone. The orthogonalityalone planner works with a surprisingly good success rate on a variety of physical objects using only a single exposure from a depth camera and no object models whatsoever. Principal axes of an object are identified from the point cloud, the approach angle is determined from the second principal axis, and the orientation of the hand is vertical from the first axis. When this technique is applied to $\mathbf{1 9}$ novel objects presented in front of a physical robot, utilizing automatic object segmentation, the grasp success rate is $\mathbf{9 8 . 4 \%}$.
\end{abstract}

\section{INTRODUCTION}

Grasping everyday objects without failure is a required feature for assistive robotics. For many applications, grasping and transportation of objects, but not fine manipulation, is required. Many elegant models have been used to assure stable grasps in the past, often including orthogality among other considerations, as in [1]. Recent work has demonstrated the power of simpler methods as well, including compliant mechanisms [2], the use of simple models [3], and real-time real-robot encounters with everyday novel objects [4], [5].

Inspired by human grasp solutions [6], we explore the use of orthogonality alone, and demonstrate grasping and picking up a surprising variety of novel objects. This has implications for the tradeoff between complexity and performance for grasping. We hope that this result will serve as a baseline for simple grasping, and underscore the importance of simple grasp properties when they are embedded in more complex grasping schemes.

Grasp testing and evaluation has often been performed in simulation [7]-[9]. Simulation testing has several clear advantages over physical testing. The high repeatability provided by computer controlled environments permits the comparison of algorithms without adding the complexity of a robotic manipulation system. Additionally, full 3D object model information in simulation permits modeling of contact forces and wrench spaces, leading to quality measures [10], [11] which allow optimization of these quantities (see [12]

E. Rombokas and Y. Matsuoka are with the Neurobotics Laboratory, Computer Science \& Engineering, University of Washington, Seattle, USA, \{rombokas, yoky\}acs. washington. edu

Peter Brook and Joshua R Smith are with the Sensor Systems Research Group, Computer Science \& Engineering, University of Washington, Seattle, USA $\{$ pbrook, jrs $\}$ @ CS. washington.edu
[11] ). Because of this, simulation has is a good evaluation tool and a great place to validate a grasp before executing it with a physical robot.

However, recent work in [6] suggests that simulationbased grasp measures might not correlate well with physical robot grasp performance. Implementing an algorithm on a real robot with real objects is integral to fully appreciating its abilities and weaknesses. Even if object models and physics simulations are perfect, the task of synching between world and simulation is complicated by the notoriously difficult problem of machine perception. When dealing with contacts [13], the dynamic consequences of misestimating distance are profound and nonlinear.

There are a variety of methods for real-world grasping. In [4] and [14], grasps were planned using supervised learning of images labeled with good grasping locations. In [5], images are used to learn grades for potential grasps based on physical properties of the grasp, such as resistance to rotation. There is also a wealth of successful work [3], [15] [17] employing vision for grasping, demonstrated on physical systems.

Point clouds have also been used successfully for grasp planning, from using learning based on labeled examples [18], allowing shape approximation via minimum volume bounding boxes [8], [19], on-the-fly object modeling [20], etc. In much of the work, point cloud sensing is used to reconstruct models of objects in simulation in order to use model-based planning methods [21], sometimes including considerations of gripper geometry [22]. In [23], heuristics based on a segmented point cloud are used in conjunction with contact-reactive grasping.

Our approach also uses a point cloud from a single depth camera exposure, however, we seek to explore the limits of open-loop grasping based on a single heuristic. Our design is driven by two motivations. First, we are motivated by the fact that single degree-of-freedom, no-intelligence prosthetic hands can grasp almost all objects of the right size and weight. This may be due to the fact that the human wearing it determines the approach angle and orientation. So rather than focusing on the individual finger movements, contact point location and properties, or object models, our approach focuses on the approach angle and orientation of the robotic hand alone and let the robot grasp and conform to the object. Second, experiments have revealed that humans approach an object orthogonal to the object principal axis [6] and approach orientation is a powerful predictor of successful grasp [3]. Our approach uses this orthogonality as the only feature used in the planner. While other grasp planning 
research has included considerations of orthogonality, our experiment suggests that this single feature can achieve a variety of grasps at considerably reduced complexity. We present this technique treating an object as one structure in Section II, and treating it as multiple subcomponents using an autonomous segmentation algorithm in Section III. Section IV discusses the implications of our experiment.

\section{ORthogonal GRASP Planner}

\section{A. Platform}

Our platform for implementation and testing was a 7 DOF Barrett WAM Arm with 4 DOF BarrettHand [24], mounted on a Segway RMP base. The base did not move around during this experiment. The platform was controlled using the Robot Operating System (ROS) [25], which provides a framework for control of the robot and acquiring measurements from the depth sensor. The system employed a BiRRT path planner [26] integrated into OpenRAVE [27] to generate trajectories from the resting position to the grasp. This path planner avoids robot self-collisions and collisions with the environment. Depth data was recorded with a Primesense depth camera providing a point cloud of 3D points. The transformation between the coordinate frame of the cloud data and that of the robot was known. The points which represent the object were segmented from the environment by defining a workspace bounding region. Points inside the region are considered part of the object, and points outside of the region are excluded.

\section{B. Methods}

1) The Orthogonality Feature: The point cloud was loaded into OpenRAVE, the principal axes were computed as shown in Fig. 1, and the midpoint of the cloud was identified. To set the approach angle, the angle between the hand $\mathrm{Z}$ axis and the second principal axis was computed and the hand was rotated so that the two axes were parallel. The hand $\mathrm{Y}$ axis was aligned with the object's first principal axis, resulting in the hand being aligned with all axes of the object as pictured in Fig. 1. The palm was translated along the second principal axis to a distance $l$ from the object midpoint, as calculated in Algorithm 1.

The distance from the palm to the object is a function only of the hand geometry and the projection of the points onto the object principal axes, not of any explicit model of the object surface. If the robot cannot achieve this grasp due to the kinematics of the arm, then the relative order of axis importance is switched so that the grasp approaches along a different axis.

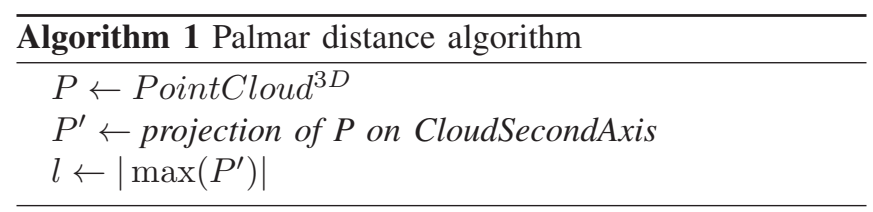

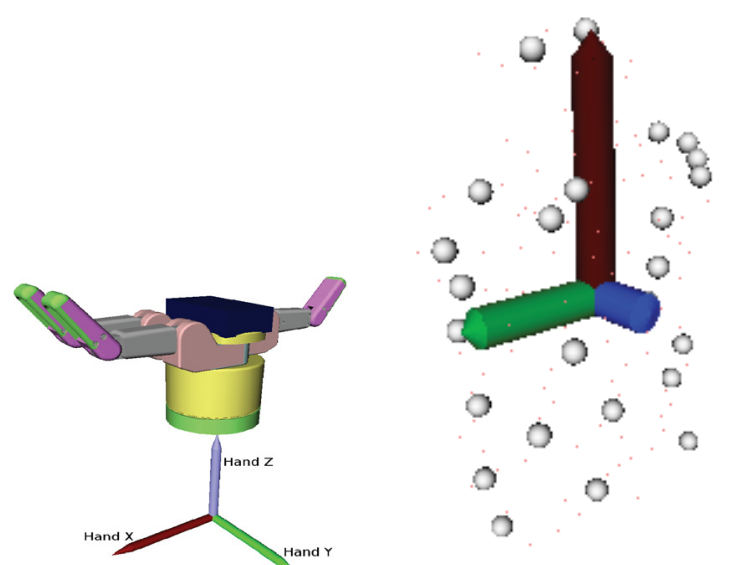

(a)

(b)

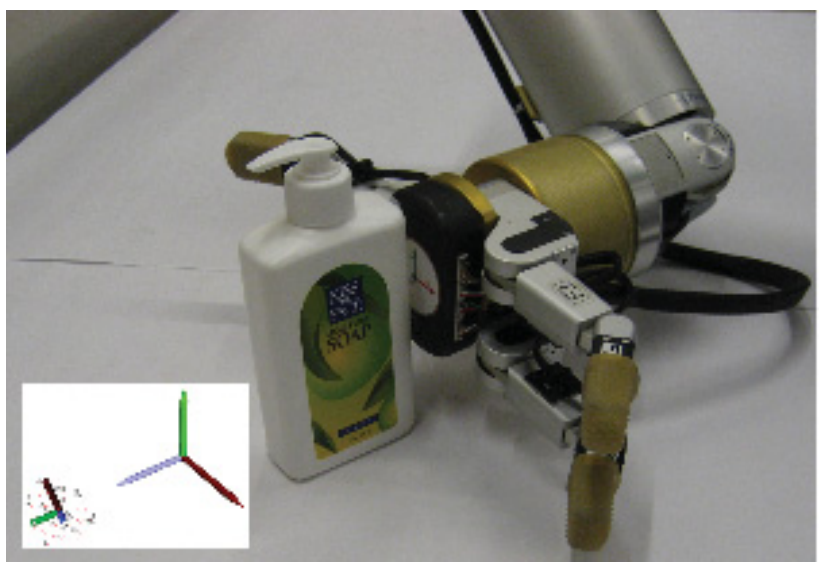

(c)

Fig. 1. (a) Hand coordinate frame. (b) Point cloud principal component axes. The lengths indicate their relative importance. (The magnitude of their associated eigenvalues.) (c) Soap grasp after alignment. The inset depicts the principal components of the point cloud on the left and the hand coordinate axis on the right.

TABLE I

OBJECTS AND THEIR CORRESPONDING INDICES

\begin{tabular}{c|c}
\hline Index & Object \\
\hline 1 & L-Shaped Wood \\
\hline 2 & C-Shaped Wood \\
\hline 3 & Pitcher \\
\hline 4 & Tea Box \\
\hline 5 & Soap Bottle \\
\hline 6 & Camera Pouch \\
\hline 7 & Plush Man \\
\hline 8 & Wine Glass \\
\hline 9 & Tripod \\
\hline 10 & Slingshot \\
\hline 11 & Wide Box \\
\hline 12 & Generic Box \\
\hline 13 & Phone \\
\hline 14 & Tall Box \\
\hline 15 & Powerstrip \\
\hline 16 & U-Shaped Wood \\
\hline 17 & Tri-Corner Wood \\
\hline 18 & Full Beer Can \\
\hline 19 & Coil of Wire \\
\hline
\end{tabular}




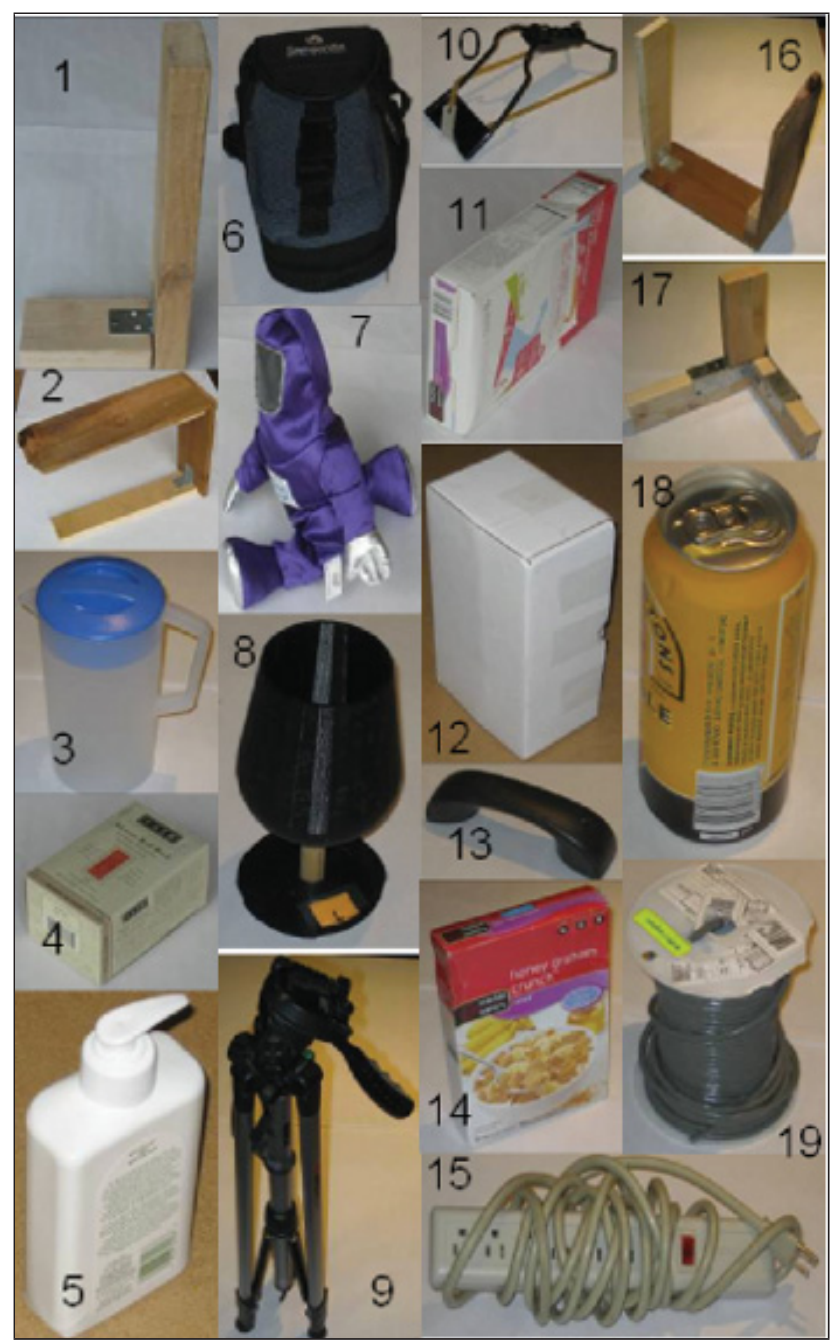

Fig. 2. All tested objects

\section{Grasp Testing}

The orthogonal grasp planner was tested on 19 objects shown in Fig. 2 with names listed in Table I. These objects ranged from the mundane, such as cans and pitchers, to more exotic objects like the slingshot. We designed four cases (objects 1, 2, 16, and 17) to create difficulties for the algorithm. These were explicitly constructed to have appendages, making it difficult to specify a single principal axis. Each object was placed on a table in front of the robot, and was tested twice at five randomly selected orientations, for a total of ten trials per object. Orientation in the table plane was randomly selected, and objects with significantly varying axis components in the axis perpendicular to the table were tested as separate objects. For instance, object 16 , the "U-Shaped Wood," is considered distinct from object 2, the "C-Shaped Wood." All objects considered were of appropriate size for the Barrett Hand, such that pinching or caging grasps would not be required. We address this choice in section IV-C.

The testing procedure was as follows:

1) A random orientation was chosen for the object.

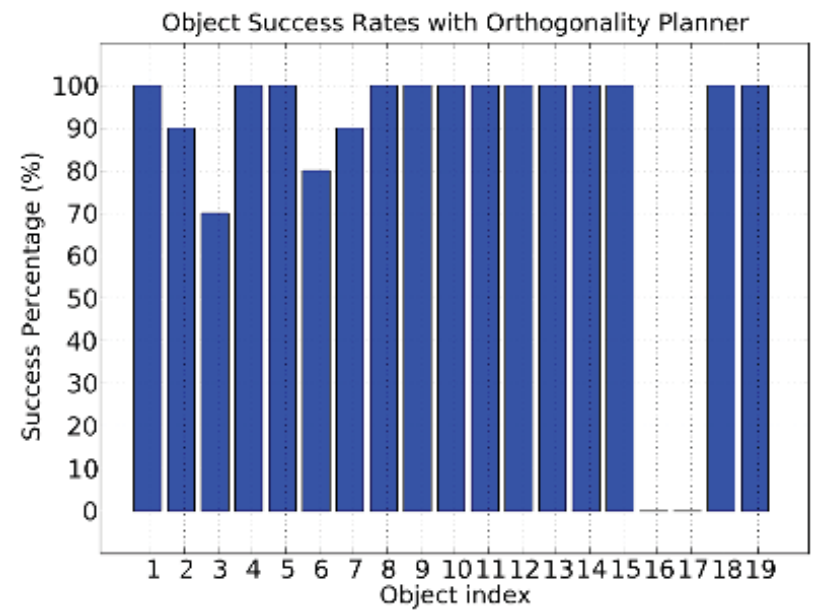

Fig. 3. Success rates by object for grasps generated by the orthogonal planner. The planner shows success for most of the objects, but is unable to generate successful grasps on two objects with exaggerated orthogonal subcomponents.

2) An exposure was taken from the depth sensor.

3) The planner calculated the principal axes.

4) The planner calculated the final grasp pose.

5) Path planner computed a collision-free trajectory to the final pose.

6) The arm moved the hand to that location.

7) The hand grasped the object by closing all three fingers rapidly and simultaneously, and continued to apply consistent torques to keep the fingers in place.

8) The object was raised up and lowered down over the course of ten seconds.

During the final grasp pose calculation, if no inverse kinematic solution could be found due to the arm configuration, the trial was aborted. If the object fell or was not successfully grasped, the trial was deemed a failure.

\section{Results}

Fig. 3 shows results on individual objects, and Fig. 4 shows typical grasp selections on two of the objects. Out of 19 objects, 13 objects were grasped with $100 \%$ success rate. The strategy appeared consistent with approaches humans would choose and showed very high robustness. There were four objects (objects 2, 3, 6, and 7) that ranged from 70\% to $90 \%$ success. The grasps chosen for 2,3 , and 7 were occasionally confounded by the object subcomponents, and object 6 was forced to use top-down pinching-type grasps due to the kinematic constraint of the object lying close to the table, which prevented the success rate from reaching $100 \%$. Interestingly, objects 16 and 17 had $0 \%$ success rate. The next section discusses these objects and addresses multipart objects.

\section{Componentwise Orthogonal Grasp Planner}

\section{A. Motivation for Componentwise Planner}

We constructed objects $1,2,16$, and 17 because these objects do not have any material to grasp at the centroid 

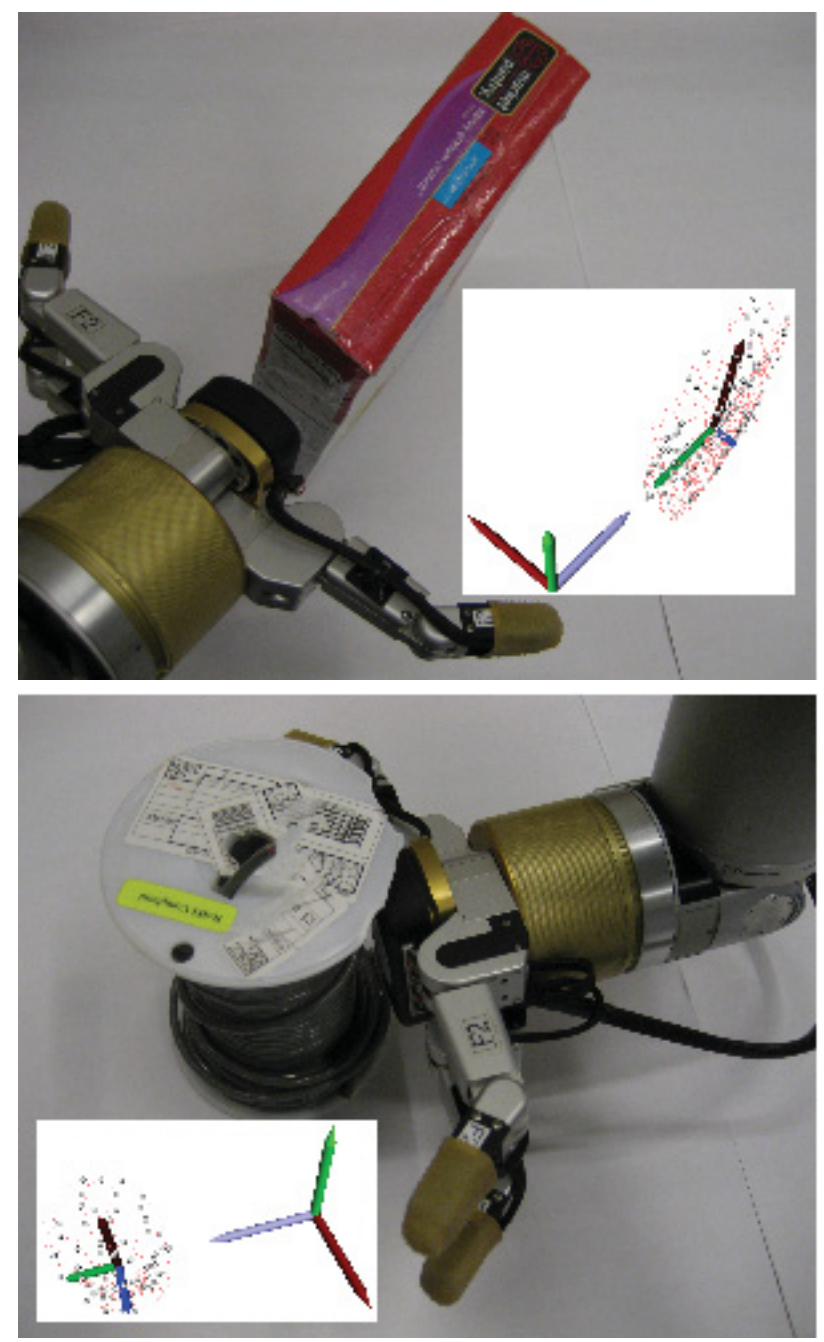

Fig. 4. Grasps chosen by orthogonal planner before finger closure. Inset are the the point clouds and object and hand axes.

of the object. There are surprisingly few objects like this in everyday environments, but we wanted to push the boundary of our algorithm by creating these objects. Object 1 was grasped with $100 \%$ success rate. This is due to an interesting aspect of approaching from the secondary principal axis: the "foot" at the bottom of the object skews the principal axis away from the main object body, but this skew is not significant in the second principal axis since the palm will be translated to be near the object extents (See Algorithm 1.) The grasps chosen this way are less robust, however, because their approach is not as perfectly orthogonal, and for this reason Object 2 was grasped with $90 \%$ success instead of $100 \%$. While object 16 is the same object as object 2, this object could not be handled at all in the configuration that is specified as object 16. Fig. 5 shows how the failure occurred. This is because their global axes differ too much from the axes of their subcomponents. If those objects could be partitioned into subcomponents and the decision of which subcomponent to grasp could be made, the grasp success could be dramatically improved.
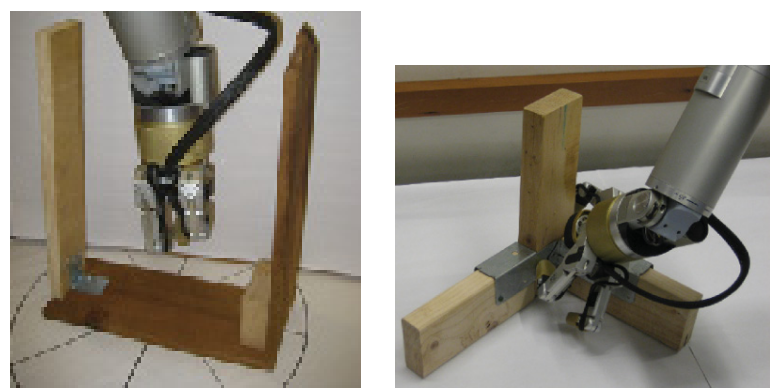

Fig. 5. Simple orthogonality-based grasps for objects 16 and 17. Highly non-convex objects have midpoints and axes which sometimes foil straightforward orthogonality grasps.

Partitioning an object into subcomponents is a wellarticulated problem in grasping, and impressive headway has been made in solving it [19], [28]. We demonstrate a simple method for detecting and accommodating these cases which prioritizes the importance of the principal axis.

\section{B. Methods}

In order to detect and use object subcomponents, we employ a method inspired by RANSAC [29]. RANSAC seeks to reject outliers by iteratively drawing subsets of the data and fitting a model to them. The model may be thought of as a cost function on the subset and fitting means parameters are found which minimize the cost. By examining the resultant models, the model which best explains the inlier data can be estimated.

For this paper, the model is the principal axis, so subsets of the point cloud are iteratively drawn and fit to a 3D line. The 3D line is the model which corresponds to the principal axis of the subset. For the results reported here, each subset contained 3 points, and 60 subsets were evaluated, but the algorithm is not critically sensitive to these values. See [30] for a review of some of the issues surrounding RANSAC performance. Those models which are highly similar are merged, and each point in the cloud is said to be "captured" by the model which best explains it in a squared-error sense. The model considered the best is the one capturing the most points, and those models which capture fewer than $1 / 4$ of that number are removed. This threshold is a free parameter which controls the degree to which a small object subcomponent should be segmented from its neighbors. The process is repeated until no model is eliminated. An example of the performance of this object subcomponent segmentation is pictured in Fig. 6.

The points captured by a model share a principal axis which well-characterizes them, but this axis may intersect other object subcomponents and capture spurious points. These points contribute error to the estimation of the subcomponent midpoint, and are removed via a self-tuning spectral clustering algorithm [31]. This clean-up of the cloud is fast, robust, and contributes no free parameters. We consider only the cloud corresponding to the best model, which is now ready for grasping by the original orthogonal grasp planner. 


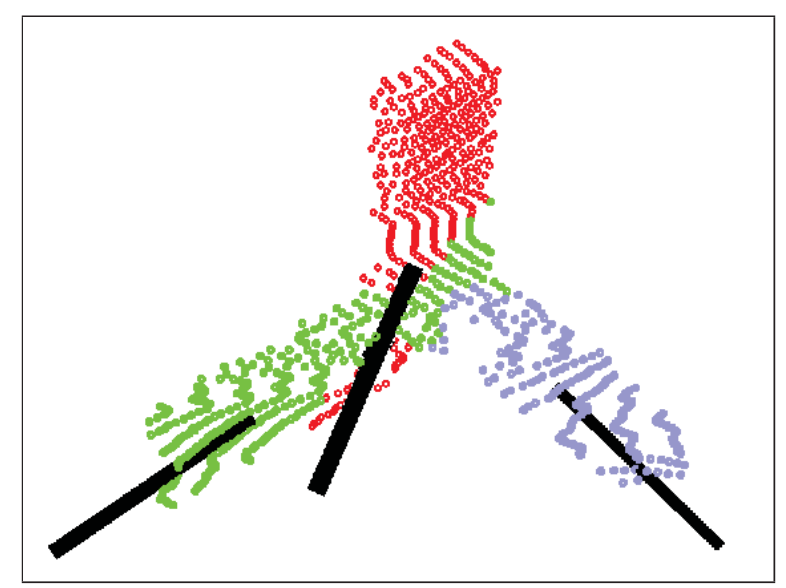

Fig. 6. Segmentation of a point cloud from object 17 into three subcomponents based on principal axis. The black lines depict the model axis which best describes each of the object subcomponents.

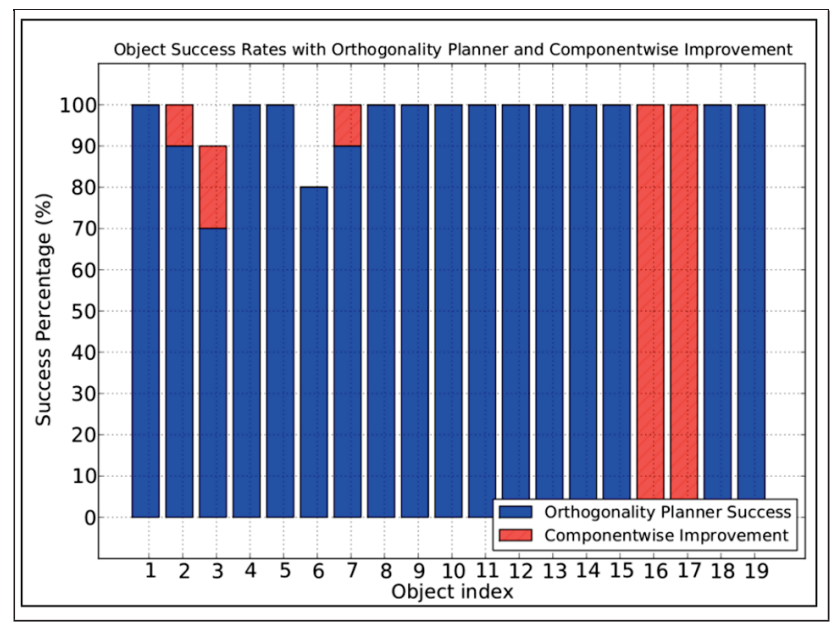

Fig. 7. Improvements from componentwise segmentation

\section{Componentwise Orthogonal Planner Results}

Segmentation of the object into components sharing a set of principal axes greatly improves grasp success. Fig. 7 shows the results with the componentwise orthogonal planner. Objects 16 and 17 were grasped with $100 \%$ success with strategies similar to the ones shown in Fig. 8. The performance for the objects that had less than $100 \%$ success rate has also improved, and the overall success rate of our algorithm was $98.4 \%$.

\section{Discussion}

\section{A. Insight From Human Solutions}

Our use of the orthogonality feature was inspired by experiments with humans [6], [32]. Often, it is perceived that drawing inspiration from biological agents adds more complexity but it is not necessarily the case that complex behaviors require complex mechanisms [33]. We are interested in using complex biological behavior to extract simple rules that help produce more robust robotic behavior. There are examples of this approach in vision [34], force control
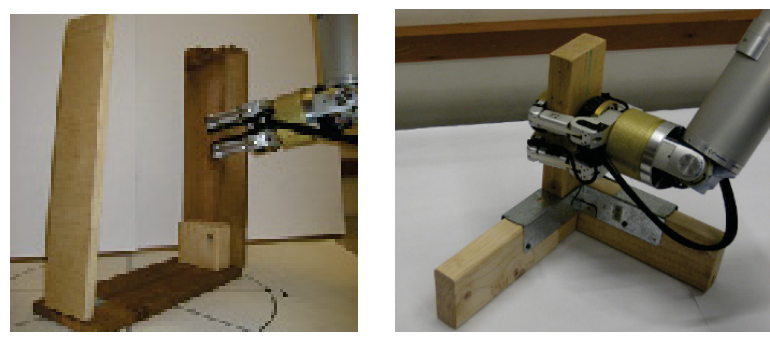

Fig. 8. Grasp correction using subcomponent segmentation for objects 16 and 17.

[35], [36], and microsurgery [37]. There could of course be uniquely robotic approaches to these same tasks, but humans serve as an existence proof for task realizability, and if robotic systems use solutions resembling those of humans they will be more able to operate in human contexts and spaces. It is not necessary to replicate general human cognitive capability to take advantage of particular capabilities with limited scope. There is some evidence that neural systems choose among a toolbox of special-purpose cognitive tools themselves [38].

\section{B. Effective Grasping with Minimal Information}

Our successful result serves as a proof of concept that useful subsets of the grasping problem can be solved with no learning, example databases, or high-dimensional feature spaces. This result does not preclude the utility of those tools; grasp planners which leverage a memory of objects and relate their observations to them could be more capable of surmounting sensing failures. The orthogonality planner is dependent on a relatively strong correlation between point cloud geometry and object geometry, but point cloud registration and object-from-environment segmentation are problems for which solutions are becoming quite good, even in the unsupervised case [39].

\section{Grasping All Objects}

Our selection of objects spans a significant variety. However, there are some object classes that present a challenge in finding valid kinematic solutions given the specific robotic hand shape. For our experiments, we avoided using objects that are too small to be grasped by the BarrettHand power grasps (even if a human operated it with a remote control). This is due to several factors including the hand size, finger configurations, and finger/palm degrees of freedom. If an object is close to other objects, such as in cluttered environments [18], [40], approach vectors to the object will be limited and the hand's size can result in collisions that would prevent grasp success.

\section{Testing Paradigms and Success Rates}

We claim the success rate of $98.4 \%$ by grasping the object and moving it up and down for 10 seconds, which is suitable for some tasks but unsuitable for others. If the grasped objects must be used in-hand, or are delicate, then the simple lifting metric used here is inadequate. The planning 
limitations of the orthogonality heuristic would be much more apparent under more demanding constraints.

\section{CONCLUSION}

A grasp planner based only on the orthogonality feature on $3 \mathrm{~d}$ point clouds can grasp a variety of objects on a physical robotic platform. This method requires no previous experience or complex object models and accommodates a wide variety of objects. This result demonstrates that exploiting insights from human behavior can result in robust grasping results with relatively little computation, and provides a baseline for comparison of more sophisticated algorithms.

\section{REFERENCES}

[1] A. Miller, S. Knoop, H. Christensen, and P. Allen, "Automatic grasp planning using shape primitives," in Robotics and Automation, 2003. Proceedings. ICRA'03. IEEE International Conference on, vol. 2. IEEE, 2003, pp. 1824-1829.

[2] A. Dollar and R. Howe, "Towards grasping in unstructured environments: Grasper compliance and configuration optimization," Advanced Robotics, vol. 19, no. 5, pp. 523-543, 2005.

[3] J. Bohg and D. Kragic, "Grasping Familiar Objects Using Shape Context," in International Conference on Advanced Robotics, Munich, Germany, June 2009.

[4] A. Saxena, J. Driemeyer, and A. Y. Ng, "Robotic grasping of novel objects using vision," Int. J. Rob. Res., vol. 27, no. 2, pp. 157-173, 2008.

[5] I. Kamon, T. Flash, and S. Edelman, "Learning to grasp using visual information," in Robotics and Automation, 1996. Proceedings., 1996 IEEE International Conference on, vol. 3, Apr 1996, pp. 2470-2476 vol.3.

[6] R. Balasubramanian, L. Xu, P. Brook, J. Smith, and Y. Matsuoka, "Human-guided grasp measures improve grasp robustness on physical robot," in Robotics and Automation, 2010. ICRA 2010. IEEE International Conference on, 2010.

[7] A. Miller and P. Allen, "Graspit! a versatile simulator for robotic grasping," vol. 11, no. 4, pp. 110-122, Dec. 2004.

[8] S. Geidenstam, K. Huebner, D. Banksell, and D. Kragic, "Learning of 2D grasping strategies from box-based 3D object approximations," in Proceedings of Robotics: Science and Systems, Seattle, USA, June 2009.

[9] Y. Liu, G. Starr, J. Wood, and R. Lumia, "Spatial grasp synthesis for complex objects using model-based simulation," Industrial Robot: An International Journal, vol. 32, no. 1, pp. 24-31, 2005.

[10] C. Ferrari and J. Canny, "Planning optimal grasps," in Robotics and Automation, 1992. Proceedings., 1992 IEEE International Conference on, May 1992, pp. 2290-2295 vol.3.

[11] A. Miller and P. Allen, "Examples of 3d grasp quality computations," in Robotics and Automation, 1999. Proceedings. 1999 IEEE International Conference on, vol. 2. IEEE, 1999, pp. 1240-1246.

[12] A. Bicchi and V. Kumar, "Robotic grasping and contact: a review," in Robotics and Automation, 2000. Proceedings. ICRA 'O0. IEEE International Conference on, vol. 1, 2000, pp. 348-353 vol.1.

[13] E. Todorov, "A convex, smooth and invertible contact model for trajectory optimization," in Robotics and Automation (ICRA), 2011 IEEE International Conference on. IEEE, 2011, pp. 1071-1076.

[14] A. Saxena, L. Wong, M. Quigley, and A. Ng, "A vision-based system for grasping novel objects in cluttered environments," in International Symposium of Robotics Research. Citeseer, 2007.

[15] P. Sanz, A. Del Pobil, J. Inesta, and G. Recatala, "Vision-guided grasping of unknown objects for service robots," in Robotics and Automation, 1998. Proceedings. 1998 IEEE International Conference on, vol. 4, May 1998, pp. 3018-3025 vol.4.

[16] D. Bowers and R. Lumia, "Manipulation of unmodeled objects using intelligent grasping schemes," vol. 11, no. 3, pp. 320-330, June 2003.

[17] A. Morales, P. Sanz, A. del Pobil, and A. Fagg, "Vision-based threefinger grasp synthesis constrained by hand geometry," Robotics and Autonomous Systems, vol. 54, no. 6, pp. 496-512, 2006.

[18] A. Saxena, L. L. S. Wong, and A. Y. Ng, "Learning grasp strategies with partial shape information," in AAAI'08: Proceedings of the 23rd national conference on Artificial intelligence. AAAI Press, 2008, pp. 1491-1494.
[19] K. Huebner, S. Ruthotto, and D. Kragic, "Minimum volume bounding box decomposition for shape approximation in robot grasping," in Robotics and Automation, 2008. ICRA 2008. IEEE International Conference on, May 2008, pp. 1628-1633.

[20] M. Krainin, P. Henry, X. Ren, and D. Fox, "Manipulator and object tracking for in-hand 3d object modeling," The International Journal of Robotics Research, 2011.

[21] B. Wang, L. Jiang, J. LI, and H. Cai, "Grasping unknown objects based on 3d model reconstruction," in Advanced Intelligent Mechatronics. Proceedings, 2005 IEEE/ASME International Conference on, July 2005, pp. 461-466.

[22] Y. Jiang, S. Moseson, and A. Saxena, "Efficient grasping from rgbd images: Learning using a new rectangle representation." ICRA, 2011.

[23] K. Hsiao, S. Chitta, M. Ciocarlie, and E. Jones, "Contact-reactive grasping of objects with partial shape information," in Intelligent Robots and Systems (IROS), 2010 IEEE/RSJ International Conference on. IEEE, 2010, pp. 1228-1235.

[24] "http://www.barrett.com/."

[25] M. Quigley, K. Conley, B. Gerkey, J. Faust, T. B. Foote, J. Leibs, R. Wheeler, and A. Y. Ng, "Ros: an open-source robot operating system," in International Conference on Robotics and Automation, ser. Open-Source Software workshop, 2009.

[26] J. Kuffner, J.J. and S. LaValle, "Rrt-connect: An efficient approach to single-query path planning," in Robotics and Automation, 2000. Proceedings. ICRA 'OO. IEEE International Conference on, vol. 2, 2000, pp. 995-1001 vol.2.

[27] R. Diankov and J. Kuffner, "Openrave: A planning architecture for autonomous robotics," Robotics Institute, Carnegie Mellon University, Tech. Rep. CMU-RI-TR-08-34, Jul. 2008.

[28] M. Richtsfeld and M. Vincze, "Point Cloud Segmentation Based on Radial Reflection," September 2009.

[29] M. Fischler and R. Bolles, "Random sample consensus: A paradigm for model fitting with applications to image analysis and automated cartography," 1981.

[30] R. Subbarao and P. Meer, "Beyond RANSAC: User independent robust regression," in Proceedings of the 2006 Conference on Computer Vision and Pattern Recognition Workshop, 2006, p. 101.

[31] L. Zelnik-Manor and P. Perona, "Self-tuning spectral clustering," Advances in neural information processing systems, vol. 17, no. 16011608, p. 16, 2004.

[32] R. H. Cuijpers, J. B. J. Smeets, and E. Brenner, "On the relation between object shape and grasping kinematics," J Neurophysiol, vol. 91, no. 6, pp. 2598-2606, 2004.

[33] V. Braitenberg, Vehicles. Mit Press, 1984.

[34] L. von Ahn, R. Liu, and M. Blum, "Peekaboom: a game for locating objects in images," in CHI '06: Proceedings of the SIGCHI conference on Human Factors in computing systems. New York, NY, USA: ACM, 2006, pp. 55-64.

[35] F. Valero-Cuevas, F. Zajac, and C. Burgar, "Large index-fingertip forces are produced by subject-independent patterns of muscle excitation," Journal of Biomechanics, vol. 31, no. 8, pp. 693-704, 1998.

[36] G. Westling and R. Johansson, "Factors influencing the force control during precision grip," Experimental Brain Research, vol. 53, no. 2, pp. 277-284, 1984.

[37] R. Kumar, P. Berkelman, P. Gupta, A. Barnes, P. S. Jensen, L. L. Whitcomb, and R. H. Taylor, "Preliminary experiments in cooperative human/robot force control for robot assisted microsurgical manipulation," in Proceedings of the IEEE International Conference on Robotics and Automation, 2000, pp. 610-617.

[38] S. Grillner, J. Hellgren, A. Ménard, K. Saitoh, and M. Wikstr "om, "Mechanisms for selection of basic motor programs-roles for the striatum and pallidum," TRENDS in Neurosciences, vol. 28, no. 7, pp. 364-370, 2005.

[39] J. Modayil and B. Kuipers, "Bootstrap learning for object discovery," in IEEE/RSJ Int. Conf. on Intelligent Robots and Systems. Citeseer, 2004, pp. 742-747.

[40] D. Berenson and S. Srinivasa, "Grasp synthesis in cluttered environments for dexterous hands," in Robotics: Science and Systems Workshop-Robot Manipulation: Intelligence in Human Environments, 2008. 